\title{
Gut microbiota and sirtuins in obesity-related inflammation and bowel dysfunction
}

Shaheen E Lakhan ${ }^{1 *}$ and Annette Kirchgessner ${ }^{1,2}$

\begin{abstract}
Obesity is a chronic disease characterized by persistent low-grade inflammation with alterations in gut motility. Motor abnormalities suggest that obesity has effects on the enteric nervous system (ENS), which controls virtually all gut functions. Recent studies have revealed that the gut microbiota can affect obesity and increase inflammatory tone by modulating mucosal barrier function. Furthermore, the observation that inflammatory conditions influence the excitability of enteric neurons may add to the gut dysfunction in obesity. In this article, we discuss recent advances in understanding the role of gut microbiota and inflammation in the pathogenesis of obesity and obesity-related gastrointestinal dysfunction. The potential contribution of sirtuins in protecting or regulating the circuitry of the ENS under inflamed states is also considered.
\end{abstract}

\section{Introduction}

Obesity has dramatically increased during the past decades and has now reached epidemic proportions in both developed and developing countries. Even in Japan where the self-reported prevalence of obesity has remained consistently low over the last 30 years, obesity is now increasing in middle-aged adults and children $[1,2]$ partly due to a western-style change in diet. The increase in obesity is associated with corresponding increases in type 2 diabetes, hypertension, cardiovascular disease and cancer [3]. Obesity is also associated with an increased incidence of gastrointestinal (GI) disorders [4] suggesting effects on the enteric nervous system (ENS), which controls virtually all gut functions (for review see [5]).

It is generally accepted that obesity is characterized by a low-grade chronic inflammation [6] in which proinflammatory cytokines play a pivotal role. The source of the inflammation is regarded as the adipose tissue itself. The adipose tissue of obese individuals, including adolescents, has been shown to produce higher levels of tumor necrosis factor (TNF)- $\alpha$ and interleukin (IL)- 6 compared to lean individuals [7-10]. In animal models of diet-induced and genetic obesity increased production of IL-1, IL-6, TNF- $\alpha$ and Toll-like receptor (TLR) signaling in adipose tissue has also been reported [11-13]. In this review, the cause of the inflammation has been

\footnotetext{
* Correspondence: slakhan@gnif.org

${ }^{1}$ Global Neuroscience Initiative Foundation, Los Angeles, CA, USA

Full list of author information is available at the end of the article
}

reevaluated and the GI tract as a potential source of inflammation and the role of gut microbiota are explored.

A high fat (HF) diet not only modulates the release of inflammatory mediators from adipocytes but also has a major impact upon the gut microbiota [14], the trillions of bacteria that normally reside within the human GI tract and upon fermentation of non-digestible carbohydrates generate short-chain fatty acids [15] and promote their absorption and storage as fat $[16,17]$. HF feeding in mice induced a low-grade inflammatory tone that was associated with changes in gut microbiota towards a decreased number of bifidobacteria [18-20], a group of bacteria, which has been shown to reduce lipopolysaccharide (LPS) levels in mice and to improve mucosal barrier function [21-23]. Interestingly, feeding obese mice with prebiotics and changing gut microbiota in favor of the Bifidobacterium spp. led to a significant improvement of gut permeability that correlated with lower portal plasma LPS levels and inflammatory tone (i.e., decreased circulating cytokines) $[18,21]$. The leakage of gut microbiota-derived LPS into the portal blood is a well-established mechanism of metabolic endotoxemia that triggers liver inflammation and oxidative stress $[17,24]$. The gut microbiota differs in composition between lean and obese individuals [14,25]. Moreover, alterations in gut microbiota seen in morbidly obese subjects are modulated by weight loss due to calorie restriction (CR) [26] or gastric bypass surgery and are

\section{( Biomed Central}


correlated with a reduction in inflammatory state $[27,28]$.

This article will discuss recent advances in understanding the role of gut microbiota and inflammation in the pathogenesis of obesity and obesity-related GI dysfunction. Since the silent information regulator (SIR) genes (sirtuins) are protective against obesity-induced inflammation and mediate, at least in part, the beneficial effects of CR (for review see $[29,30]$ ), the potential contribution of sirtuin signaling in the bowel under inflamed states is also considered.

\section{Gut microbiota}

The human GI tract is dominated by anaerobic bacteria belonging to three bacterial phyla: Firmicutes, Bacteroidetes, and Actinobacteria [31]. Greater than $90 \%$ of the microbiota in a normal distal gut is represented by the Bacteroidetes and Firmicutes phyla [32]. The Firmicutes, which is the largest bacterial phylum, comprises over 200 genera of predominantly Gram-positive bacteria, including Lactobacillus, Mycoplasma, Bacillus, and Clostridium species. The Bacteroidetes phylum is composed of three large classes of Gram-negative bacteria: Cytophaga, Flavobacterium, and Bacteroidales. Members of the Bacteroidetes and Firmicutes phyla have been shown to be influenced by HF feeding and obesity [32-34]. The Actinobacterium phylum consists of Gram-positive bacteria and includes the genus Bifidobacterium, which is increased upon consumption of prebiotics, indigestible carbohydrates, which stimulate the growth of particular species of the microflora (for review see [35]).

Initial observations to suggest that the gut microbiota contribute to obesity were prompted by the observations of Gordon and colleagues [16,36]. Studies in leptin-deficient $o b / o b$ mice showed a different proportion of the two dominating divisions, Bacteroidetes and Firmicutes compared to lean wild-type (WT) mice. Compared with lean littermates fed the same polysaccharide-rich diet, obesity was associated with a $50 \%$ reduction in Bacteriodetes and a proportional division-wide increase in Firmicutes in obese mice. To definitively demonstrate that gut microbiota composition is a cause and not a consequence of obesity, cecal microbiota from lean and obese mice were transplanted into the gut of germ-free (GF) mice. GF mice, raised from birth in sterile conditions, are significantly thinner than microbially colonized mice, despite eating the same amounts of food. Furthermore, after 2 weeks, conventionalization of GF mice with the cecal microbiota from normal mice produced a $57 \%$ increase in total body fat, a 2.3 -fold increase in hepatic triglycerides, and much higher levels of leptin production and insulin resistance which was not dependent on an increase in chow consumption or changes in energy expenditure $[37,38]$. Evidence was obtained to suggest that an increased energy harvest from the diet contributed to obesity in host GF mice. This might be due to an increase in energy extraction due to bacterial fermentation of polysaccharides and also to the ability of the gut microbiota to upregulate fasting-induced adipose factor, a circulating lipoprotein lipase, which increases cellular uptake of fatty acids and triglyceride accumulation in adipocytes. Shotgun metagenomic analysis of the gut microbiome in obese and lean mice revealed an enrichment of genes involved in energy harvest in obese mice [34,38]. These included genes involved in sensing and degrading dietary polysaccharides, transporters of the resulting mono- and oligosaccharides, and genes involved in their intracellular metabolism. Thus, the microbiome of obese mice had increased fermentation capabilities resulting in increased levels of short chain fatty acids (SCFAs) in the cecum [38]. Similarly, the gut microbiome of obese individuals has increased fermentation capacity resulting in elevated SCFA production [38]. Interestingly, mice deficient in either of the SCFA receptors are leaner than their wildtype counterparts [39], further implicating SCFAs in the development of obesity. Currently, there is no consensus as to whether the gut microbiota plays a causative role in obesity or is modulated in response to the obese state itself or the diet in obesity. Further studies, especially on the regulatory role of SCFA in human energy homeostasis are needed to clarify the physiological consequences of an "obese style" microbiota and any putative dietary modulation of associated disease risk.

Data from human studies were generally consistent with the results from animal models. The first study describing qualitative changes of the gut microbiota in human obese subjects was published a few years ago [40]. In this study, the analysis of fecal samples of obese versus matched lean individuals showed a shift in bacterial phyla (lower Bacteroidetes and more Firmicutes). Interestingly the authors observed that after weight loss (following a fat restricted or a carbohydrate restricted low calorie diet) the ratio of Bacteroidetes to Firmicutes approached a lean type profile after 52 weeks [40]. In addition, humans who have undergone gastric bypass surgery for morbid obesity, have a microbial composition that is different from both obese and slim individuals [41]. A metagenomic study which included monozygotic and dizygotic twins concordant for leanness or obesity and their mothers, also showed that obesity was associated with a markedly reduced bacterial diversity, a relative depletion of Bacteroidetes, and a higher proportion of Actinobacteria [42].

The hypothesis of more specific modulation of the gut microbiota community in obesity (instead of those obtained at the wide phylum levels) is supported by several studies. Children with developmentally unusual gut 
microbiota appear to have predispositions to obesity [43] and another study found that the response of overweight adolescents to a diet and exercise weight-loss program was dependent on the initial gut microbiota prior to the treatment [26]. Moreover, differences in fecal microbiota were shown to predict overweight in children during the first year in life [43]. In a prospective study, Bifidobacteria spp. number was higher in children who exhibited a normal weight at 7 years than in children developing overweight. Moreover, they observed that the Staphylococcus aureus were lower in children who maintain a normal weight than in children becoming overweight several years later. The authors proposed that $S$. aureus may act as a trigger of lowgrade inflammation, contributing to the development of obesity [43]. In agreement with these last findings, significant differences have been observed in gut microbiota composition according to the body weight gain during pregnancy [44]. They also found that the Bifidobacterium genus was present in higher numbers in normal-weight than in overweight women and also in women with lower weight gain during pregnancy [44]. The Bifidobacterium genus was also poorly represented in the fecal samples of diabetic patients compared with healthy individuals [45].

Similar results linking gut microbiota to obesity have been described in models of diet-induced obesity. In mice, ingestion of a HF diet resulted in an increase of Firmicutes and this was transmissible to lean GF recipient mice [34]. In addition, an increase in Bacteroidales and Clostridiales was found in rats fed a HF diet regardless of whether they exhibit either an obesity-prone or obesity-resistant phenotype. However, an increase in Enterobacteriales was seen in the microbiota of obesityprone rats only [17]. Gut inflammation has been shown to promote the growth of Enterobacteriaceae [46]; therefore, an increase in this family may be a consequence of gut inflammation in the HF obesity prone mice rather than a cause. Taken together, these data demonstrate that it is the consumption of a HF diet rather than obesity that accounts for the change in the gut microbiota, but it is the development of inflammation that is associated with the appearance of hyperphagia and an obese phenotype.

Although humans are interested in manipulating microbiota to aid in weight loss, the food industry has been engaged for decades in manipulating microbiota to increase in weight gain through the use of low-dose antibiotics, usually called antibiotic growth promoters (AGPs) as feed additives [47]. Evidence from the food industry has shown that antibiotics, such as avoparcin (a vancomycin analogue) and Firmicute probiotics (e.g., Lactobacillus and Enterococcus) that modify the microbiota can act as growth promoters increasing the size and weight of farm animals [48]. Notably, a recent human clinical study showed significant weight gain can occur in humans after a six-week intravenous treatment of vancomycin plus gentamycin for infective endocarditis with a risk of obesity. Lactobacillus sp, a microorganism intrinsically resistant to vancomycin was found at higher concentration in the feces of obese patients [49]. In contrast, the absence of specific microbiota or its almost complete reduction with broad-spectrum antibiotics prevents or reverses HF-induced obesity $[36,50]$. Treatment with rifaximin (Xifaxan ${ }^{\circledR}$, Salix Pharmaceuticals, Morrisville, NC, USA), a nonsystemic rifamycinderived antibiotic that exhibits low gastrointestinal absorption while retaining potent antibacterial activity [51] for two weeks has recently been shown to provide significant relief of symptoms associated with IBS, such as bloating, abdominal pain, and loose or watery stools [52], which are also observed in obese individuals. Modulation of the gut microbiota with antibiotic therapy has been reported in obese mice. In addition, antibiotics reversed insulin resistance improving glycemic control [50].

\section{Gut inflammation and barrier function}

It is now well established that obesity is an inflammatory condition and that "low grade chronic" inflammation, associated with insulin and leptin resistance exists in obese individuals [6]. The source of the inflammation is commonly regarded as the adipose tissue itself, which is known to produce inflammatory mediators [53]. However, the gut microbiota could also be a potential source of inflammation.

A HF diet is associated with the expression of two inflammatory biomarkers in the intestine, TNF- $\alpha$ and nuclear factor kappa B (NF- $\kappa \mathrm{B})$. The presence of gut bacteria is required for the induction of TNF- $\alpha$ and NF$\kappa \mathrm{B}$ since GF mice given a similar diet did not exhibit up-regulation of these pro-inflammatory markers [54]. Moreover, the observation that increases in intestinal TNF- $\alpha$ precede yet significantly correlate with body weight gain, body fat, and subsequent development of insulin resistance, supports a potential role of gutderived TNF- $\alpha$ in the development of HF-induced obesity and obesity-related disease [54].

Recent work has shown that gut microbiota can initiate the inflammatory state of obesity through the activity of LPS, part of the outer membrane of Gramnegative bacteria that is released into the gut lumen when bacteria die. Cani et al. [18] reported that mice fed a HF diet present a chronic increase in circulating LPS, which they called "metabolic endotoxemia". The level of serum LPS is increased by about twice in obese, diabetic, or high-fat fed individuals, by processes involving an increase in chylomicron formation, a decrease in 
gut barrier integrity, and a decrease in alkaline phosphatase activity, which is the enzyme responsible for the cleavage of the LPS in the intestine $[55,56,21]$.

LPS can trigger the inflammatory process by binding to the CD14 toll-like receptor-4 (TLR4) complex in the gut wall. When metabolic endotoxemia was reproduced by subcutaneous infusion of LPS, animals developed the same metabolic abnormalities induced by a HF diet, while LPS knock out (CD14-/-) mice were resistant to the effects of both HF diet and LPS infusion [57]. Moreover, chronic (4 week) administration of LPS in mice causes hyperphagia and an increase in adiposity and metabolic changes seen with ingestion of HF diet $[21,57]$. In a subsequent experiment, changes in gut microbiota composition induced by antibiotic treatment reduced the cecal content of LPS and improved measures of inflammation, such as macrophage infiltration of adipose tissue, closely correlating with an improvement in the obese phenotype in both HF fed and $o b / o b$ mice [19].

Activation of TLR4 causes the secretion of IL- 6 and TNF- $\alpha$, supporting the role of LPS in triggering the downstream inflammatory processes associated with obesity, such as metabolic disease [20,57]. Ingestion of a $\mathrm{HF}$ diet induced a significant postprandial elevation of LPS, accompanied by an increased mononuclear cell expression of TLR-4, NF-KB and suppressor of cytokine signaling-3 (SOCS-3), an adipokine involved in insulin resistance [58].

Interestingly, mice genetically deficient in TLR-5 have an altered gut microbiota composition that correlates with obesity and several features of the metabolic syndrome including hyperlipidemia, hypertension, and insulin resistance, which could at least in part be attributed to increased food consumption [59]. In contrast to the $o b / o b$ mouse model of obesity, which is characterized by a phylum-level shift in Bacteroidetes and Firmicutes, the TLR5-deficient mice exhibit altered abundance in over one-hundred specific bacterial phylotypes. A direct causal relationship between the altered gut microbiota and obesity was demonstrated by gut microbiota transplants where GF mice receiving the gut microbiota from TLR5-deficient mice gained significantly more weight compared to mice that received gut microbiota from wildtype mice [59]. These results support the emerging view that the gut microbiota contributes to obesity and suggest that malfunction of the innate immune system may promote the development of obesity-related disorders such as metabolic syndrome.

Mucosal barrier function is maintained by several interrelated systems, including mucous secretion, chloride and water secretion, and binding together of epithelial cells at their apical junctions by tight junction (TJ) proteins. The disruption of the TJ complex leads to leakage of water and proteins into the lumen, as described in relapsing diarrhea, and to the translocation of intraluminal solutes, such as bacterial endotoxins (LPS), into the system circulation [60]. Activation of TLR4 has previously been shown to alter the TJ complex and increase intestinal permeability [61]. Modulation of gut bacteria following a HF diet strongly increases intestinal permeability, by reducing the expression of genes coding for two intestinal TJ proteins, $\mathrm{ZO}$ 1 and occluding [19]. Alteration in occludin distribution has also been reported in vitro on epithelial cells stimulated with pro-inflammatory cytokines; thus, occludin was chosen as a marker of TJ disruption [24,62].

Since aberrant gut microbiota and a "leaky" mucosal barrier are found in obesity they offer potential targets for intervention that would include modulation of the intestinal microbiota to correct an imbalance, as well as tightening of interepithelial junctions. Enhancement of barrier function by probiotic bacteria has been observed both in in vitro models and in vivo in the whole animal [63]. Probiotics are live microorganisms that have a beneficial effect on the intestinal mucosa via several proposed mechanisms that include inhibition of the mucosal adhesion of pathogens, improvement of the barrier function of the epithelium, and alteration of the immune activity of the host. They may also regulate intraluminal fermentation and stabilize the intestinal microbiota [64]. Probiotic bacteria are Lactobacilli spp., certain types of Streptococcus, and Bifidobacteria spp., but also other non-pathogenic bacilli such as E. coliNisle 1917 and yeasts such as Saccharamyces boulardii. They secrete short chain fatty acids, an action that results in decreased luminal $\mathrm{pH}$ and production of bactericidal proteins. Butyric acid, a byproduct of bacterial fermentation of fiber, has been shown to nourish colonic enterocytes, enhancing mucosal integrity [65].

Researchers have demonstrated the utility of probiotics for obesity in HF fed mice $[57,66]$, which is associated with a decrease in the number of Bifidobacteria [21]. An increase in Bifidobacteria in $o b / o b$ mice was associated with a significant improvement of gut permeability measured in vivo; this improvement was linked to an increase in TJ mRNA expression and protein distribution [21]. In addition, the rise in Bifidobacteria was correlated with a decrease in plasma LPS concentrations and therefore, a significant reduction in markers of oxidative and inflammatory stress [21]. Potential beneficial effects of probiotics on gut motility via a direct action on the ENS or epithelial cells have also been demonstrated [67]. In experimental studies, Lactobacillus inhibited post-infective intestinal hypercontractility through an unidenfied, heat-labile fermentation-product and by blocking calcium-dependent potassium channels [68,68,69]. Supernatant of Escherichia coli Nissle 1917 
enhanced human colonic motility in vitro and acute exposure of colonic mucosa to Lactobacillus rhamnosus GG significantly reduced the acetylcholine-stimulated human colonic contractions in a dose- and time-dependent manners [70]. Administration of L. reuteri altered the motility of ex vivo colonic segment of rat; it decreased the amplitudes of contractions and increased intraluminal fluid filling pressure thresholds for evoking pressure pulses [71]. Overall, probiotics will likely have an emerging therapeutic role in preventing and treating obesity and obesity-related inflammation.

\section{Gut dysfunction and obesity}

Many obese individuals report symptoms suggestive of gut dysfunction including bloating, abdominal pain and diarrhea $[4,72,73]$. Bloating and upper abdominal pain increased in frequency with increasing body mass index (BMI). There was also a significant positive relationship between BMI and diarrhea. In contrast, no significant relationship was observed between BMI and constipation, even though it was more frequent in obese patients [4]. Potential mechanisms to explain the increased bowel frequency would be rapid gastric emptying, which has been reported in some groups of obese patients [74], and increased colonic motility, although the latter has not yet been demonstrated.

Obesity is associated with gastroesophageal reflux disease (GERD), nonalcoholic fatty liver disease (NAFLD), and increased occurrence of cholelithiasis [75,76]. GERD has been shown to be more common in obese patients than in those with a BMI within normal range, and an increase in the BMI above the 95 percentile for age and gender is a significant risk factor for GERD $[77,78]$. Also, a higher BMI is associated with more frequent and more severe heartburn and regurgitation in patients with GERD and increasing BMI is a strong predictor of heartburn during sleep $[79,80]$.

In patients with irritable bowel syndrome (IBS), heartburn was more likely to be present in subjects with obesity, and epigastric pain and nausea, were also more common in overweight patients with IBS. However, in an adjusted log linear model, no significant interaction was found between BMI and any other studied symptom and heartburn was found to be independent of IBS [81].

An important and well described correlation also exists between obesity and colorectal cancer [82]. Epidemiologic data have shown that obesity independently increases colorectal cancer risk, particularly in males, but the mechanisms are poorly understood [83]. Serum leptin level in colon cancer patients who were overweight or obese were significantly higher compared to patients with normal weight [84]. mRNA levels of the novel inflammatory factors lipocalin-2, chitinase-3 like- 1 and osteopontin are increased in human visceral adipose tissue of individuals with colon cancer [85]. Leptin upregulates pro-inflammatory cytokines in discrete cells within the mouse colon [86]. IL6, IL1 $\beta$ and CXCL1 were upregulated by leptin and localized to discrete cells in gut epithelium, lamina propria, muscularis and at the peritoneal serosal surface.

Diet-induced weight loss in obese individuals reduces colorectal inflammation and greatly modulates inflammatory and cancer-related gene pathways [87]. After weight-loss, rectosigmoid biopsies showed a $25-57 \%$ reduction in TNF- $\alpha$, IL-1 $1 \beta$, and MCP-1 concentrations. Gene arrays showed dramatic down-regulation of proinflammatory cytokine and chemokine pathways, prostaglandin metabolism, oxidative stress pathways and the transcription factor CREB. These data imply that obesity is accompanied by inflammation in the colorectal mucosa and that weight loss reduces this inflammatory state and may thereby lower colorectal cancer risk [87].

Obesity predicts persistence of abdominal pain in children with functional gastrointestinal disorders [88]. Obese children (mean age 13 years) were more likely to have abdominal pain, higher intensity of pain, higher frequency of pain, school absenteeism and disruption of daily activities than non-obese children [88]. Obesity is more common in children with celiac disease, a T cellmediated chronic autoimmune enteropathy occurring in genetically susceptible individuals, and manifested by a permanent intolerance of gluten-containing products [89]. The most common presenting symptoms among obese patients were abdominal pain, diabetes, and diarrhea. Symptoms improved in all patients on a glutenfree diet.

\section{Role of the ENS}

Under both physiological and pathological conditions, the ENS, the intrinsic innervation of the bowel, regulates intestinal mucosal function and coordinates the activity of the GI tract. The ENS is a component of the autonomic nervous system with the unique ability to function independently from the CNS (for review, see [90]). Enteric ganglia are organized into two major ganglionated plexuses, namely the myenteric (Auerbach's) and submucosal (Meissner's) plexus, and contain a variety of functionally distinct neurons, including primary afferent neurons, interneurons, and motor neurons, synaptically linked to each other in microcircuits. While the myenteric plexus mainly regulates intestinal motility, the submucosal plexus together with nerve fibers in the lamina propria are involved in regulating epithelial transport. These nerves form networks within the lamina propria of both crypts and villi with the terminal axons in close contact with the basal lamina, an ideal position not only to affect epithelial cell functions but also to detect absorbed nutrients and antigens. Substances released 
from epithelial cells may act on nerve terminals to change the properties of enteric neurons and cause peripheral sensitization. Consequently, permanent or even transient structural alterations in the ENS disrupt normal GI function.

The ENS is increasingly recognized as a regulatory housekeeper of the epithelial barrier integrity, in addition to its ascribed immunomodulatory potential (for review see [5]). Inflammation affects both epithelial integrity and barrier function and, in turn, loss of barrier function perpetuates the inflammatory condition. Several studies have demonstrated structural changes within enteric ganglia in gut inflammation (see [91,92] for review). For example, damage to axons has been observed in the inflamed human intestine in episodes of inflammatory bowel disease (IBD) [93]. Other changes that occur in the ENS during inflammation include altered neurotransmitter synthesis, content, and release, changes in glial and myenteric cell numbers and a myenteric ganglionitis associated with infiltrates of lymphocytes, plasma cells and mast cells [94-96]. In fact, experimental data show that gut inflammation, even if mild, could lead to persistent changes in GI nerve and smooth muscle function, resulting in dysmotility, hypersensitivity, and dysfunction (for review see $[91,92]$ ). Furthermore, alterations in gut function were observed even after the resolution of an acute intestinal inflammation [97-99]. Thus, the breakdown of mucosal barrier function as occurs in obesity could cause alterations in the patterns of gut motility, abnormal secretion, and changes in visceral sensation that contributes to symptom generation. In a rodent model of diet-induced obesity the secretomotor function of submucosal neurons was compromised, which may lead to an altered host defense with a resultant change in intestinal flora contributing to the maintenance of obesity [100]. The breakdown of mucosal barrier function may at least partially explain the link between obesity and gut dysfunction. Whether the persistent alterations in GI motility observed in many obese patients are due to inflammation-related changes in the properties of enteric nerves is not known. However, probiotic lactic acid producingbacteria have been shown to prevent and alleviate GI disturbances and to normalize the cytokine profile which might be of an advantage for patients suffering from obesity [101].

\section{Role of sirtuins}

In the past decade, a novel class of regulators, the silent regulator 2 (SIR2), has been linked to metabolic regulation and aging and shown to mediate CR-induced longevity in yeast and possibly other organisms (for review see [29]). Mammalian sirtuins are conserved with seven genes (SIRT1-7) homologous to the yeast Sir2 gene.
Like their yeast homologs, the mammalian sirtuins are class III histone deacetylases and require $\mathrm{NAD}(+)$ as a cofactor to deacetylate substrates ranging from histones to transcriptional regulators. The nuclear sirtuins (SIRT1, SIRT6, and SIRT7), the mitochondrial sirtuins (SIRT3, SIRT4, and SIRT5), and the cytosolic sirtuin (SIRT2) regulate diverse metabolic functions. For example, SIRT6 functions in genomic stability and transcriptional control of glucose metabolism and its deficiency (SIRT6-/-) causes a lethal hypoglycemia [102,103]. SIRT6 is highly expressed in the CNS and mice overexpressing SIRT6 are protected against diet-induced obesity [104]. In contrast, neural-specific SIRT6 knockout mice become obese during adult life [105], further highlighting the importance of SIRT6 in the context of nutrient metabolism. SIRT3 is an integral regulator of mitochondrial function and its depletion results in hyperacetylation of critical mitochondrial proteins that protect against hepatic lipotoxicity under conditions of nutrient excess. Livers of mice fed on a HF diet had reduced SIRT3 activity [106].

The beneficial effect of CR on aging and various metabolic disorders is dependent on the activation of SIRT1 and can be mimicked by resveratrol, a product present in grape skin and red wine, which activates the SIRT1 enzyme $[107,108]$. Mild to moderate red wine consumption has anti-inflammatory properties, and can reduce the risk of cardiovascular disease and cancer. The resveratrol content in red wine is often cited to account for this "French paradox." Evidence for a role of sirtuins in obesity comes from emerging understanding of the regulatory role sirtuins play in metabolic pathways and adaptations linked with obesity and aspects of metabolic syndrome. These include the expression of adipocyte cytokines (adipokines), the maturation of fat cells, insulin secretion, modulation of plasma glucose levels, cholesterol and lipid homeostasis and mitochondrial energy capacity [29].

SIRT1, for example, is involved in regulating the expression of adipokines such as adiponectin and TNF$\alpha$, has been linked to hypothalamic control of energy balance, plays a role in adipogenesis, and is involved in the regulation of lipolysis and fatty acid mobilization in response to fasting.[29] Evidence from animal studies in which sirtuins are under- or over-expressed, and from limited human evidence, also suggest a role for sirtuins in obesity. Existing evidence on resveratrol suggests that this compound may have sirtuin-mediated anti-obesity effects [109].

Fasting leads to the up-regulation of SIRT1 in adipose tissue of mice, pigs and humans whereas decreased SIRT1 expression is associated with obesity [29]. In both $d b / d b$ and obese HF fed mice SIRT1 expression is low in adipose tissue [110]. Circumstances that result in 
SIRT1 under-expression in adipose tissue enhanced adipogenesis, while circumstances that promote fat SIRT1 over-expression were characterized by attenuated adipogenesis and increased lipolysis [111]. Consistent with this idea, lean women had more than twofold higher SIRT1 expression compared to obese women [112]. Benefits of SIRT1 over-expression also included less inflammation, better glucose tolerance, and almost complete protection against hepatic steatosis, suggesting that SIRT1 plays an important role in obesity-associated metabolic adverse effects. Consequently, if activation of SIRT1 results in loss of body fat without decreasing caloric intake, this could open the door for novel treatment and prevention strategies for obesity and related diseases.

Genetic variation in SIRT1 is related to BMI and risk of obesity in humans $[113,114]$. In a recent Belgian case/control study of 1,068 obese patients (BMI $\geq 30$ $\mathrm{kg} / \mathrm{m}^{2}$ ) and 313 normal weight control subjects, a SIRT1 single nucleotide polymorphism (SNP) associated with visceral obesity parameters in obese men but not women [113]. In two large and independent Dutch Caucasian populations, two common variants in SIRT1 were associated with lower BMI. Carriers of these two common genetic variants had 9-11\% decreased risk of being overweight and 13-18\% decreased risk of being obese compared with noncarriers [114].

SIRT1 has recently been implicated in the regulation of obesity-related inflammation. Zhu et al. [115] demonstrated that resveratrol, a SIRT1 activator, decreased TNF- $\alpha$-induced MCP-1 secretion in 3T3-L1 adipocytes. Pfluger et al. [116] showed over-expression of SIRT1 in mice resulted in a lower level of IL- 6 and TNF- $\alpha$ in the serum of transgenic mice fed a HF diet and an attenuated response to TNF- $\alpha$-induced NF- $\kappa \mathrm{B}$ activation in transgenic mouse embryonic fibroblasts. Resveratrol regulates human adipocyte number and down-regulates the expression and secretion of IL- 6 and IL- 8 from mammalian adipocytes in a SIRT1-dependent manner [117]. Furthermore, mice fed a diet supplemented with $0.4 \%$ resveratrol for 10 weeks showed significantly lower body weight and visceral fat-pad weights than HF diet fed mice. Resveratrol significantly attenuated the HF dietinduced up-regulation of a number of pro-inflammatory cytokines such as TNF- $\alpha$ and IL- 6 , and their upstream molecules, including TLR4 and NF- $\kappa$ B in epididymal adipose tissues of mice [12]. Thus, increased SIRT1 activity appears to be anti-inflammatory in mice and resveratrol may improve obesity-induced inflammation and add to the potential of this dietary polyphenol in the control of obesity. In contrast, inhibition of SIRT1 appears to be pro-inflammatory. Studies using small interfering RNA (siRNA) to knock down SIRT1 reported an increase in TNF- $\alpha$-induced MCP-1 and other pro- inflammatory genes in 3T3-L1 adipocytes [118]. Taken together, these data demonstrate that a decrease in SIRT1 activity increases activation of NF- $\kappa \mathrm{B}$ and transcription of pro-inflammatory mediators. These results have important clinical implications and may thus provide a valuable new strategy for treatment of obesity and its related diseases.

In addition to adipose tissue, SIRT1 is highly expressed in the hypothalamus where it appears to be involved in regulating energy homeostasis, food intake and body weight $[108,119]$. Fasting up-regulates hypothalamic SIRT1 expression, which is associated with a fasting-induced increase in hunger, and presumably part of the complex adaptations against CR-induced weight loss [119]. Conversely, pharmacological inhibition of hypothalamic SIRT1 decreases food intake and body weight gain in rodents [120], suggesting that hypothalamic SIRT1 decreases food intake and body weight gain in rodents. Lack of SIRT1 in pro-opiomelanocortin (POMC) neurons causes hypersensitivity to HF obesity [121].

SIRT1 is also expressed in the gut where it exerts antiinflammatory effects in acute intestinal inflammation and suppresses intestinal tumorigenesis and colon cancer associated with colitis [122-124]. Rats fed with $1 \mathrm{mg}$ of resveratrol $/ \mathrm{kg} /$ day (a human equivalent dose) for 25 days, and in the last five days, $5 \%$ DSS to induce colitis, displayed increased lactobacilli and bifidobacteria as well as a reduced increase in enterobacteria upon DSS treatment. In addition, resveratrol significantly protected the colonic mucosa architecture, reduced body weight loss, diminished the induced anemia and reduced systemic inflammatory markers possibly via the down-regulation of NF- $\kappa \mathrm{B}$ [124]. Also, resveratrol up-regulated SIRT1 expression in the mucosa and mitigated the increase in the number of mucosal CD4+ T cells suggesting that resveratrol may exert its anti-inflammatory effects by modulating activated immune cells [123]. Thus, activation of SIRT1 maintains gut barrier function, which is compromised in obesity [125] and through its regulation of gut inflammation controls colitis and colon cancer, which are also more prevalent in obese individuals [126].

The ENS also contains sirtuins. We have shown for the first time that neurons in the murine colon display SIRT1 immunoreactivity (Figure 1). The cellular localization of SIRT1 is predominantly nuclear and displayed by neurons in both the submucosal and myenteric plexus consistent with areas strongly compromised by aging [127]. These findings propose a role for SIRT1 in gut motility and secretion and suggest a previously unrecognized role of enteric SIRT1 in regulating energy homeostasis. Moreover, activation of enteric sirtuin pathways could offer a therapeutic approach to treat 


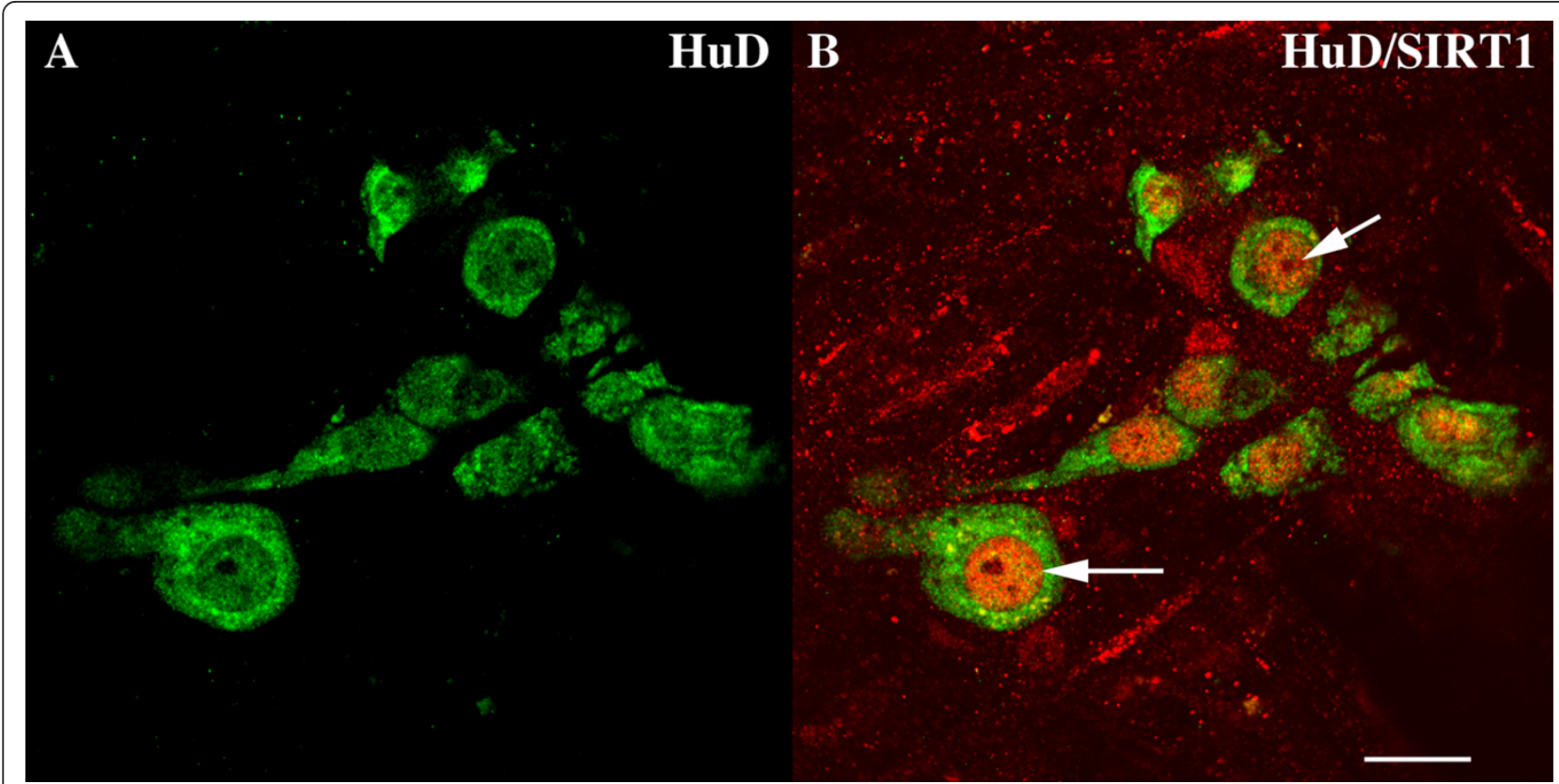

Figure 1 Immunohistochemical localization of the class III histone deacetylase SIRT1 (Sir2) in the murine enteric nervous system. A Confocal image of a whole mount preparation of colon stained with a goat antibody to the neuronal marker human neuronal protein (HuD; 1:100; Santa Cruz; sc-5977; green). HuD immunoreactivity is displayed by neurons in a myenteric ganglion. B. Double label confocal image of the same area depicted in $\mathbf{A}$ stained with an antibody to HuD and a SIRT1-specific antibody made in rabbit (1:500; Abcam Inc. Cambridge MA; ab 16640). Myenteric neurons display both HuD (green) and nuclear SIRT1 immunoreactivity (red). For whole-mount preparations, segments of colon were cut along the mesenteric border and the resulting sheet of gut was pinned flat, mucosal side up, in a silicone elastomer (Sylgard, Dow Corning, Midland, MI)-coated dish. The tissue was fixed for 3 hours with 4\% paraformaldehyde in $0.1 \mathrm{M}$ phosphate buffer (pH 7.4). After fixation, the preparations were washed in phosphate-buffered saline (PBS) for 1 hour and whole-mount preparations of longitudinal muscle with adherent myenteric plexus (LMMP) were generated as previously described [128]. Non-specific binding was blocked by incubating the preparations with 6\% (v/v) normal horse serum, with Triton X-100 (0.5\%), in PBS for 60 minutes. The preparations were then exposed for $24 \mathrm{~h}$ to primary antibodies at $4^{\circ} \mathrm{C}$. After washing with PBS, sites of bound primary antibodies were detected by incubation with donkey anti-rabbit or donkey anti-goat secondary antibodies coupled to DyLightTM 549 (1:400; Jackson ImmunoResearch Labs.West Grove, PA) or DyLightTM 488 (1:400; Jackson ImmunoResearch Labs.) for 3 hours. Confocal images were obtained using an Olympus FluoView FV300 confocal microscope. Scale bar, $30 \mu \mathrm{m}$

obesity-related gut dysfunction. Clearly, further research is required to explore the role of sirtuin proteins in enteric neurobiology during normal and inflamed states.

\section{Conclusions}

Obesity is considered a major public health concern globally as it predisposes to a number of chronic human diseases. Recent studies report an aberrant gut microbiota in obese individuals and that gut microbial metabolic activities, especially fermentation can impact on a number of mammalian physiological functions linked to obesity. Those data suggest that specific changes in the gut microbiota characterize the obese state and associated metabolic diseases, including diabetes. Gut microbiota, which affect barrier function also modulate the activity of the ENS, a key player in gut dysfunction. Since aberrant gut microbiota and a "leaky" mucosal barrier are found in obesity they offer potential targets for intervention that would include modulation of the intestinal microbiota to correct an imbalance, as well as tightening of interepithelial junctions. However, we may not conclude, from the papers published until now, that targeting one specific bacterial target is sufficient to get an improvement of a complex disease such as obesity. Future studies using newly developed techniques to evaluate the gut microbiota in obese patients and/or animal models of obesity are certainly needed. In addition, future research should focus on the role of sirtuin proteins in the gut and their function in obesity and gut dysfunction. Studies suggest that resveratrol acts as an anti-inflammatory agent in the gut by targeting the SIRT1 gene. Thus, resveratrol may be a therapeutic target against obesity-related inflammation and gut dysfunction.

\section{List of abbreviations}

Body mass index: BMl; calorie restriction: $C R$; enteric nervous system: ENS; gastroesophageal reflux disease: GERD; gastrointestinal: Gl; germ-free: GF; high-fat diet: HF; inflammatory bowel disease: IBD; irritable bowel syndrome: IBS; interleukin: IL; interferon: IFN; lipopolysaccharide: LPS; nonalcoholic fatty 
liver disease: NAFLD; nuclear factor kappa B: NF-KB; pro-opiomelanocortin: POMC; short chain fatty acid: SCFA; silent information regulator: SIR; silent information regulator genes: sirtuins; small interfering RNA: siRNA; suppressor of cytokine signaling-3: SOCS-3; tight junction: TJ; Toll-like receptor: TLR; tumor necrosis factor: TNF; wild type: WT.

\section{Acknowledgements}

This development of this work was supported by the Global Neuroscience Initiative Foundation (GNIF). The authors wish to extend special thanks to GNIF research assistants/associates Violeta Osegueda, Nirali Shah, and Magdalena Hofer for their suggestions and editing support.

\section{Author details}

${ }^{1}$ Global Neuroscience Initiative Foundation, Los Angeles, CA, USA. ${ }^{2}$ School of Health and Medical Sciences, Seton Hall University, South Orange, NJ, USA.

\section{Authors' contributions}

All authors participated in the preparation of the manuscript, and read and approved the final manuscript.

\section{Competing interests}

The authors declare that they have no competing interests.

Received: 10 July 2011 Accepted: 24 November 2011

Published: 24 November 2011

\section{References}

1. Matsushita Y, Yoshiike F, Yoshita K, Takimoto H: Trends in childhood obesity in Japan over the last 25 years from the national nutrition survey. Obesity Res 2004, 12:205-214.

2. Sasai H, Sairenchi T, Hiroyasu I, Irie F, Otaka E, Tanaka K, Ota H, Muto T: Relationship between obesity and incident diabetes in middle-aged and older Japanese adults: The Ibaraki prefectural health study. Mayo Clin Proc 2010, 85:36-40.

3. Field AE, Coakley EH, Must A, Spadano JL, Laird N, Dietz WH, Rimm E, Colditz GA: Impact of overweight on the risk of developing common chronic diseases during a 10-year period. Arch Intern Med 2001, 161:1581-1586.

4. Delgado-Aros S, Locke GR, Camilleri M, Talley NJ, Fett S, Zinsmeister AR, Melton LJ: Obesity is associated with increased risk of gastrointestinal symptoms: a population-based study. Am J Gastroenterol 2004, 99:1801-1806.

5. Snoek SA, Verstege MI, Boeckxstaens GE, van den Wijngaard RM, de Jonge WJ: The enteric nervous system as a regulator of intestinal epithelial barrier function in health and disease. Expert Rev Gastroenterol Hepatol 2010, 4:637-651.

6. Hotamisligil GS: Inflammation and metabolic disorders. Nature 2006, 444:860-867.

7. Kern PA, Ranganathan S, Li C, Wood L, Ranganathan G: Adipose tissue tumor necrosis factor and interleukin- 6 expression in human obesity and insulin resistance. Am J Physiol Endocrinol Metab 2001, 280:E745-E751.

8. Syrenicz A, Garanty-Bogachka B, Syrenicz M, Gebala A, Walczak M: Lowgrade systemic inflammation and the risk of type 2 diabetes in obese children and adolescents. Neuro Endocrinol Lett 2006, 27:453-458.

9. Panagiotakos DB, Pitsavos C, Yannakoulia M, Chrysohoou C, Stefanadis C: The implication of obesity and central fat on markers of chronic inflammation: The ATTICA study. Atherosclerosis 2005, 183:308-315.

10. Viser M, Bouter LM, McQuillan GM, Wener MH, Harris TB: Low-grade systemic inflammation in overweight children. Pediatrics 2001, 107:E13.

11. Brake DK, Smith EO, Mersmann H, Smith CW, Robker RL: ICAM-1 expression in adipose tissue: effects of diet-induced obesity in mice. Am J Physiol Cell Physiol 2006, 291:C1232-1239.

12. Kim SJ, Choi Y, Choi YH, Park T: Obesity activates toll-like receptormediated proinflammatory signaling cascades in the adipose tissue of mice. J Nutr Biochem 2011.

13. Xu H, Barnes GT, Yang Q, Tan G, Yang D, Chou CJ, Sole J, Nichols A, Ross JS, Tartaglia LA, et al: Chronic inflammation in fat plays a crucial role in the development of obesity-related insulin resistance. J Clin Invest 2003, 1123:1821-1830.
14. DiBaise JK, Zhang H, Crowell MD, Krajmalnik-Brown R, Decker GA, Rittmann BE: Gut microbiota and its possible relationship with obesity. Mayo Clin Proc 2008, 83:460-469.

15. Conterno LFF, Viola R, Tuohy KM: Obesity and the gut microbiota: does up-regulating colonic fermentation protect against obesity and metabolic disease? Genes Nutr 2011, 6:241-260.

16. Bäckhed F, Ding H, Wang T, Hooper LV, Koh GY, Nagy A, Semenkovich CF, Gordon Jl: The gut microbiota as an environmental factor that regulates fat storage. Proc Natl Acad Sci 2004, 101:15718-15723.

17. de La Serre CB, Ellis CL, Lee J, Hartman AL, Rutledge JC, Raybould HE: Propensity to high-fat diet-induced obesity in rats is associated with changes in the gut microbiota and gut inflammation. Am J Physiol Gastrointest Liver Physiol 2010, 299:G440-448.

18. Cani PD, Neyrinck AM, Fava F, Knauf C, Burcelin RG, Tuohy KM, Gibson GR, Delzenne NM: Selective increases of bifidobacteria in gut microflora improve high-fat-diet-induced diabetes in mice through a mechanism associated with endotoxaemia. Diabetologia 2007, 50:2374-2383.

19. Cani PD, Bibiloni R, Knauf C, Waget A, Neyrinck AM, Delzenne NM, Burcelin R: Changes in gut microbiota could control endotoxemiainduced inflammation in high-fat diet-induced obesity and diabetes in mice. Diabetes 2008, 57:1470-1481.

20. Cani PD, Delzenne NM, Amar J, Burcelin R: Role of gut microflora in the development of obesity and insulin resistance following high-fat diet feeding. Pathol Biol (Paris) 2008, 56:305-309.

21. Cani PD, Possemiers S, Van de Wiele T, Guiot Y, Everard A, Rottier O, Geurts L, Naslain D, Neyrinck A, Lambert DM, et al: Changes in gut microbiota control inflammation in obese mice through a mechanism involving GLP-2-driven improvement of gut permeability. Gut 2009, 58:1091-1103.

22. Griffiths EA, Duffy LC, Schanbacher FL, Qiao H, Dryja D, Leavens A Rossman J, Rich G, Dirienzo D, Ogra PL: In vivo effects of bifidobacteria and lactoferrin on gut endotoxin concentration and mucosal immunity in Balb/c mice. Dig Dis Sci 2004, 49:579-589.

23. Wang Z, Xiao G, Yao Y, Guo S, Lu K, Sheng Z: The role of bifidobacteria in gut barrier function after thermal injury in rats. J Trauma 2006, 61:650-657.

24. Brun P, Castagliuolo I, Di Leo V, Buda A, Pinzani M, Palu G, Martines D: Increased intestinal permeability in obese mice: new evidence in the pathogenesis of nonalcoholic steatohepatitis. Am J Physiol Gastrointest Liver Physiol 2007, 292:G518-G525.

25. Scarpellini E, Campanale M, Leone D, Purchiaroni F, Vitale G, Lauritano EC, Gasbarrini A: Gut microbiota and obesity. Intern Emerg Med 2010, 5(Suppl 1):S53-56.

26. Santacruz A, Marcos A, Wärnberg J, Martí A, Martin-Matillas M, Campoy C, Moreno LA, Veiga O, Redondo-Figuero C, Garagorri JM, et al: Interplay between weight loss and gut microbiota composition in overweight adolescents. Obesity 2009, 17:1906-1915.

27. Chaudhri O, Wynne K, Bloom SR: Can gut hormones control appetite and prevent obesity? Daibetes Care 2008, 31(Suppl 2):S284-S289.

28. Furet JP, Kong LC, Tap J, Poitou C, Basdevant A, Bouillot JL, Mariat D, Corthier G, Doré J, Henegar C, et al: Differential adaptation of human gut microbiota to bariatric surgery-induced weight loss: links with metabolic and low-grade inflammation. Diabetes 2010, 59:3049-3057.

29. Kelly G: A review of the sirtuin system, its clinical implications, and the potential role of dietary activators like resveratrol: part 2. Alt Med Rev 2010, 15:313-338.

30. Schug TT, Li X: Sirtuin 1 in lipid metabolism and obesity. Ann Med 2011.

31. Greiner T, Backhed F: Effects of the gut microbiota on obesity and glucose homeostasis. Trends Endocrinol Metab 2011.

32. Hildebrandt MA, Hoffmann C, Sherrill-Mix SA, Keilbaugh SA, Hamady M, Chen YY, Knight R, Ahima RS, Bushman F, Wu GD: High-fat diet determines the composition of the murine gut microbiome independently of obesity. Gastroenterology 2009, 137:1716-1724.

33. Backhed $\mathrm{F}$ : Changes in intestinal microflora in obesity: cause or consequence? J Pediatr Gastroenterol Nutr 2009, 48(Suppl 2):S56-S57.

34. Turnbaugh PJ, Bäckhed F, Fulton L, Gordon Jl: Diet-induced obesity is linked to marked but reversible alterations in the mouse distal gut microbiome. Cell Host Microbe 2008, 3:213-223.

35. Damaskos D, Kolios G: Probiotics and prebiotics in inflammatory bowel disease: microflora "on the scope". Br J Clin Pharmacol 2008, 65:453-467. 
36. Bäckhed F, Manchester JK, Semenkovich CF, Gordon Jl: Mechanisms underlying the resistance to diet-induced obesity in germ-free mice. Proc Natl Acad Sci 2007, 104:979-984.

37. Ley RE, Backhed F, Turnbaugh P, Lozupone CA, Knight RD, Gordon Jl: Obesity alters gut microbial ecology. Proc Natl Acad Sci 2005, 102:11070-11075.

38. Turnbaugh PJ, Ley RE, Mahowald MA, Magrini V, Mardis ER, Gordon Jl: An obesity-associated gut microbiome with increased capacity for energy harvest. Nature 2006, 444:1027-1031.

39. Bjursell M, Admyre T, Goransson M, Marley AE, Smith DM, Oscarsson J, Bohlooly-Y M: Improved glucose control and reduced body fat mass in free fatty acid recepto 2-deficient mice fed a high-fat diet. Am J Physiol Endocrinol Metab 2011, 300:E211-220.

40. Ley RE, Turnbaugh PJ, Klein S, Gordon Jl: Microbial ecology: human gut microbes associated with obesity. Nature 2006, 444:1022-1023.

41. Zhang $H$, DiBaise JK, Zuccolo A, Kudrna D, Braidotti M, Yu Y, Parameswaran P, Crowell MD, Wing R, Rittmann BE, et al: Human gut microbiota in obesity and after gastric bypass. Proc Natl Acad Sci USA 2009, 106:2365-2370.

42. Turnbaugh PJ, Hamady M, Yatsunenko T, Cantarel BL, Duncan A, Ley RE, Sogin ML, Jones WJ, Roe BA, Affourtit JP, et al: A core gut microbiome in obese and lean twins. Nature 2009, 457:480-484.

43. Kalliomaki M, Collado MC, Salminen S, Isolauri E: Early differences in fecal microbiota composition in children may predict overweight. Am J Clin Nutr 2008, 87:534-538.

44. Collado MC IE, Laitinen K, Salminen S: Distinct composition of gut microbiota during pregnancy in overweight and normal-weight women. Am J Clin Nutr 2008, 88:894-899.

45. Wu X MC, Han L, Nawaz M, Gao F, Zhang S, Yu P, et al: Molecular characterisation of the faecal microbiota in patients with type II diabetes. Curr Microbiol 2010, 61:69-78.

46. Lupp C, Robertson ML, Wickham ME, Sekirov I, Champion OL, Gaynor EC, Finlay BB: Host-mediated inflammation disrupts the intestinal microbiota and promotes the overgrowth of Enterobacteriaceae. Cell Host Microbe 2007, 2:119-129.

47. Frost AJWJ: Antibiotics and animal production. In Microbiology of Animals and Animal Products. Edited by: JB W. New York: Elsevier; 1991:181-194.

48. Lin J: Effect of antibiotic growth promoters on intestinal microbiota in food animals: a novel model for studying the relationship between gut microbiota and human obesity? Front Microbiol 2011, 2:53.

49. Thuny F R, Casalta JP, Angelakis E, Habib G, Raoult D: Vancomycin treatment of infective endocarditis is linked with recently acquired obesity. PLOS One 2010, 5:e9074

50. Membrez M, Blancher F, Jaquet M, Bibiloni R, Cani PD, Burcelin RG, Corthesy I, Macé K, Chou CJ: Gut microbiota modulation with norfloxacin and ampicillin enhances glucose tolerance in mice. FASEB 2008, 22:2416-2426.

51. Koo HL, HL DuPont: Rifaximin: a unique gastrointestinal-selective antibiotic for enteric diseases. Curr Opin Gastroenterol 2010, 26:17-25

52. Pimentel M, Lembo A, Chey WD, Zakko S, Ringel Y, Yu J, Mareya SM, Shaw AL, Bortey E, Forbes WP: Rifaximin therapy for patients with irritable bowel syndrome without constipation. NEJM 2011, 364:22-32

53. Fain J: Release of inflammatory mediators by human adipose tissue is enhanced in obesity and primarily by the nonfat cells: A review. Mediators Inflamm 2010.

54. Ding S, Chi MM, Scull BP, Rigby R, Schwerbrock NM, Magness S, Jobin C Lund PK: High-fat diet: bacteria interactions promote intestinal inflammation which precedes and correlates with obesity and insulin resistance in mouse. PLOS ONE 2010, 5:e12191.

55. Erridge C, Attina T, Spickett CM, Webb DJ: A high-fat meal induces lowgrade endotoxemia: evidence of a novel mechanism of postprandial inflammation. Am J Clin Nutr 2007, 86:1286-1292.

56. Ghanim H, Abuaysheh S, Sia CL, Korzeniewski K, Chaudhuri A, FernandezReal J, Dandona P: Increase in plasma endotoxin concentrations and the expression of toll-like receptors and suppressor of cytokine signling-3 in mononuclear cells after a high-fat, high-carbohydrate meal. Diabetes Care 2009, 32:2281-2287.

57. Cani PD, Amar J, Iglesias MA, Poggi M, Knauf C, Bastelica D, Neyrinck AM, Fava F, Tuohy KM, Chabo C, et al: Metabolic endotoxemia initiates obesity and insulin resistance. Diabetes 2007, 56:1761-1772.
58. Anderson PD, Mehta NN, Wolfe ML, Hinkle CC, Pruscino L, Comiskey LL, Tabita-Martinez J, Sellers KF, Rickels MR, Ahima RS, et al: Innate immunity modulates adipokines in humans. J Clin Endocrinol Metab 2007, 92:2272-2279.

59. Vijay-Kumar M, Aitken JD, Carvalho FA, Cullender TC, Mwangi S, Srinivasan S, Sitaraman SV, Knight R, Ley RE, Gewirtz AT: Metabolic syndrome and altered gut microbiota in mice lacking Toll-like receptor 5. Science 2010, 328:228-231.

60. Katz KD, Hollander D, Vadheim CM, McElree C, Delahunty T, Dadufalza VD, Krugliak P, Rotter Jl: Intestinal permeability in patients with Crohn's disease and their healthy relatives. Gastroenterology 1989, 97(4):927-931.

61. Gribar SC, Richardson WM, Sodhi CP, Hackam DJ: No longer an innocent bystander: epithelial toll-like receptor signaling in the development of mucosal inflammation. Mol Med 2008, 14(9-10):645-659.

62. Bruewer M, Luegering A, Kucharzik T, Parkos CA, Madara JL, Hopkins AM, Nusrat A: Proinflammatory cytokines disrupt epithelial barrier function by apoptosis independent mechanisms. J Immunol 2003, 171:6164-6172.

63. Ohland $\mathrm{CL}$, Macnaughton WK: Probiotic bacteria and intestinal epithelial barrier function. Am J Physiol Gastrointest Liver Physiol 298(6):G807-819.

64. Bixquert Jimenez M: Treatment of irritable bowel syndrome with probiotics. An etiopathogenic approach at last? Rev Esp Enferm Dig 2009, 101(8):553-564.

65. Guyonnet D, Chassany O, Ducrotte P, Picard C, Mouret M, Mercier CH, Matuchansky C: Effect of a fermented milk containing Bifidobacterium animalis DN-173 010 on the health-related quality of life and symptoms in irritable bowel syndrome in adults in primary care: a multicentre, randomized, double-blind, controlled trial. Aliment Pharmacol Ther 2007, 26:475-486.

66. Kondo S, Xiao J-Z, Satoh T, Odamaki T, Takahashi S, Sugahara H, Yaeshima T, Iwatsuki K, Kamei A, Abe K: Antiobesity effects of Bifidobacterium breve strain B-3 supplementation in a mouse model with high-fat diet-induced obesity. Biosci, Biotechnol, Biochem 2010, 74:1656-1661.

67. Kunze WA MY, Wang B, Huizinga JD, Ma X, Forsythe P, Bienenstock J: Lactobacillus reuteri enhances excitability of colonic $\mathrm{AH}$ neurons by inhibiting calcium-dependent potassium channel opening. J Cell Mol Med 2009, 13:2261-2270.

68. Ma X MY, Wang B, Huizinga JD, Bienenstock J, Kunze W: Lactobacillus reuteri ingestion prevents hyperexcitability of colonic DRG neurons induced by noxious stimuli. Am J Physiol Gastrointest Liver Physiol 2009, 296:G868-875.

69. McKeman DP FP, Dinan TG, Cryan JF: The probiotic Bifidobacterium infantis 35624 displays visceral antinociceptive effects in the rat. Neurogastroenterol Motil 2010, 22:1029-1035.

70. Bar F VKH, Roblick U, Bruch HP et al: Cell-free supernatants of Escherichia coli Nissle 1917 modulate human colonic motility; evidence from an in vitro organ bath study. Neurogastroenterol Motil 2009, 21:559-566.

71. Wang B MY, Diorio C, Wang L, Huizinga JD, Bienenstock J, Kunze W: Lactobacillus reuteri ingestion and IK(Ca) channel blockade have similar effects on rat colon motility and myenteric neurons. Neurogastroenterol Motil 2010, 22:98-107.

72. Levy RL, Linde JA, Feld KA, Crowell MD, Jeffery RW: The association of gastrointestinal symptoms with weight, diet, and exercise in weight-loss program participants. Clin Gastroenterol Hepatol 2005, 3:992-996.

73. Talley NJ, Howell S, Poulton R: Obesity and chronic gastrointestinal tract symptoms in young adults: a birth cohort study. Am J Gastroenterol 2004, 99:1807-1814.

74. Verdich C, Madsen JL, Toubro S, Buemann B, Holst JJ, Astrup A: Effect of obesity and major weight reduction on gastric emptying. Int J Obes Relat Metab Disord 2000, 24:899-905.

75. Ashburn DD, Reed MJ: Gastrointestinal system and obesity. Crit Care Clin 2010, 26:625-627

76. Moayyedi P: The epidemiology of obesity and gastrointestinal and other diseases: an overview. Dig Dis Sci 2008, 53:2293-2299.

77. Choi CW, Kim GH, Song CS, Wang SG, Lee BJ IH, Kang DH, Song GA: Is obesity associated with gastropharngeal reflux disease? World J Gastroenterl 2008, 14:265-271

78. Dore MP, Maragkoudakis E, Fraley K, Pedroni A, Tadeu V, Realdi G, Graham DY, Delitala G, Malaty HM: Diet, lifestyle, and gender in gastroesophageal reflux disease. Dig Dis Sci 2008, 53:2027-2032. 
79. Dickman R, Feroze H, Fass R: Gastroesophageal reflux disease and irritable bowel syndrome: a common overlap syndrome. Curr Gastroenterol Rep 2006, 8:261-265

80. Nocon M, Labenz J, Jaspersen D, Meyer-Sabellek W, Stolte M, Lind T, Malfertheiner P, Willich SN: Association of body mass index with heartburn, regurgitation and esophagitis: results of the progression of gastroesophageal reflux disease study. J Gastroenterol Hepatol 2007, 22:1728-1731.

81. Schmulson M, Pulido D, Escobar C, Farfan-Labone B, Lopez-Alvarenga JC: Heartburn and other related symptoms are independent of body mass index in irritable bowel syndrome. Rev Exp Enferm Dig (Madrid) 2010 102:229-233.

82. John BJ, Irukulla S, Abulafi AM, Kumar D, Mendall MA: Systematic review: adipose tissue, obesity and gastrointestinal diseases. Aliment Pharmacol Ther 2006, 23:1511-1523.

83. Bassett JK, Severi G, English DR, Bagietto L, Krishnan K, Hopper JL, Giles GG: Body size, weight change, and risk of colon cancer. Cancer Epediemiol Biomarkers Prev 2010, 19:2978-2986.

84. Stachowicz M, Mazurek U, Nowakowska-Zajdel E, Niedworok E, Fatyga E, Muc-Wierzgon M: Leptin and its receptors in obese patients with colorectal cancer. J Biol Regul Homeost Agents 2010, 24:287-295.

85. Catalán V, Gómez-Ambrosi J, Rodríguez A, Ramírez B, Silva C, Rotellar F, Hernández-Lizoain JL, Baixauli J, Valentí V, Pardo F, et al: Up-regulation of the novel proinflammatory adipokines lipocalin-2, chitinase-3 like 1 and osteopontin as well as angiogenic-related factors in visceral adipose tissue of patients with colon cancer. J Nutr Biochem 2010

86. Padidar S, Farquharson AJ, Williams LM, Kelaiditi E, N. H, Arthur JR, Drew JE: Leptin upregulates pro-inflammatory cytokines in discrete cells within mouse colon. J Cell Physiol 2010.

87. Pendyala S, Neff LM, Suarez-Farinas M, Holt PR: Diet-induced weight loss reduces colorectal inflammation: implications for colorectal carcinogenesis. Am J Clin Nutr 2010.

88. Bonilla S, Wang D, Saps M: Obesity predicts persistence of pain in children with functional gastrointestinal disorders. Int J Obes (Lond) 2010.

89. Venkatasubramani N, Telega G, Werlin SL: Obesity in pediatric celiac disease. J Pediatr Gastroenterol Nutr 2010, 51:295-297.

90. Furness J: The enteric nervous system: normal functions and enteric neuropathies. Neurogastroenterol Motil 2008, 20(Suppl 1):32-38.

91. Mawe GM SD, Sharkey KA: Plasticity of enteric nerve functions in the inflamed and postinflamed gut. Neurogastroenterol Motil 2009, 21:481-491.

92. Lakhan SE, Kirchgessner A: Neuroinflammation in inflammatory bowel disease. J Neuroinflammation 2010, 7:37

93. Geboes K, Collins S: Structural abnormalities of the nervous system in Crohn's disease and ulcerative colitis. Neurogastroenterol Motil 1998, 10:189-202.

94. Bernardini N, Segnani C, Ippolito C, De Griogio R, Colucci R, FaussonePellegrini MS, Chiarugi M, Campani D, Castagna M, Mattii L, et al: Immunohistochemical analysis of myenteric ganglia and interstitial cells of Cajal in ulcerative colitis. J Cell Mol Med 2011.

95. De Giorgio R, Camilleri M: Human enteric neuropathies: morphology and molecular pathology. Neurogastroenterol Motil 2004, 16(5):515-531.

96. Tornblom H, Lindberg G, Nyberg B, Veress B: Full-thickness biopsy of the jejunum reveals inflammation and enteric neuropathy in irritable bowel syndrome. Gastroenterology 2002, 123(6):1972-1979.

97. Hoffman JM MN, Sharkey KA, Mawe GM: The relationship between inflammation-induced neuronal excitability and disrupted motor activity in the guinea pig distal colon. Neurogastroenterol Motil 2011, 23:673.

98. Dunlop SP, Jenkins D, Spiller RC: Distinctive clinical, psychological, and histological features of postinfective irritable bowel syndrome. Am J Gastroenterol 2003, 98(7):1578-1583.

99. Isgar B, Harman M, Kaye MD, Whorwell PJ: Symptoms of irritable bowe syndrome in ulcerative colitis in remission. Gut 1983, 24(3):190-192.

100. Hyland NP, Rybicka JM, Ho W, Pittman QJ, Macnaughton WK, Sharkey KA: Adaptation of intestinal secretomotor function and nutrient absorption in response to diet-induced obesity. Neurogastroenterol Motil 2010, 22:602-e171.

101. Sullivan A, Nord CE, Evengard B: Effect of supplement with lactic-acid producing bacteria on fatigue and physical activity in patients with chronic fatigue syndrome. Nutr J 2009, 8:4

102. Mostoslavsky R, Chua KF, Lombard DB, Pang WW, Fischer MR, Gellon L LP, Mostoslavsky G, Franco S, Murphy MM, Mills KD, et al: Genomic instability and aging-like phenotype in the absence of mammalian SIRT6. Cell 2006, 124:315-329.

103. Xiao C, Kim HS, Lahusen T, Wang RH, Xu X, Gavrilova O, Jou W, Gius D, Deng CX: SIRT6 deficiency results in severe hypoglycemia by enhancing both basal and insulin-stimulated glucose uptake in mice. $J$ Biol Chem 2010, 285:36776-36784

104. Kanfi Y, Peshti V, Gil R, Naiman S, Nahum L, Levin E, Kronfeld-Schor N, Cohen HY: SIRT6 protects against pathological damage caused by dietinduced obesity. Aging Cell 2010, 9:162-173.

105. Schwer B, Schumacher B, Lombard DB, Xiao C, Kurtev MV, Gao J, Schneider Jl, Chai H, Bronson RT, Tsai LH, et al: Neural sirtuin 6 (Sirt6) ablation attenuates somatic growth and causes obesity. PNAS 2010, 107:21790-21794.

106. Kendrick AA, Choudhury M, Rahman SM, McCurdy CE, Friederich M, Van Hove JL, Watson PA, Birdsey N, Bao J, Gius D, et al: Fatty liver is associated with reduced SIRT3 activity and mitochondrial protein hyperacetylation. Biochem J 2011, 433:505-514.

107. Howitz KT, Bitterman KJ, Cohen HY, Lamming DW, Lavu S, Wood JG, Zipkin RE, Chung P, Kisielewski A, Zhang LL, et al: Small molecule activators of sirtuins extend Saccharomyces cerevisiae lifespan. Nature 2003, 425:191-196.

108. Wood JG, Rogina B, Lavu S, Howitz K, Helfand SL, Tatar M, Sinclair D: Sirtuin activators mimic caloric restriction and delay ageing in metazoans. Nature 2004, 430:686-689

109. Kim S, Jin Y, Choi Y, Park T: Resveratrol exerts anti-obesity effects via mechanisms involving down-regulation of adipogenic and inflammatory processes in mice. Biochem Pharmacol 2011.

110. Qiao I, Shao J: Sirt1 regulates adiponectin gene expression through Foxo1-C/EBPalpha transcriptional complex. J Biol Chem 2006, 281:39915-39924.

111. Picard F, Kurtev M, Chung N, Topark-Ngarm A, Senawong T, Machado De Oliveira R, Leid M, McBurney MW, Guarente L: Sirt1 promotes fat mobilization in white adipocytes by repressing PPAR-gamma. Nature 2004, 429:771-776.

112. Pedersen SB, Olholm J, Paulsen SK, Bennetzen MF, Richelsen B: Low Sirt1 expression, which is upregulated by fasting, in human adipose tissue from obese women. Int J Obes (Lond) 2008, 32:1250-1255.

113. Peeters AV, Beckers S, Verrijken A, Mertens I, Roevens P, Peeters PJ, Van Hul W, Van Gaal LF: Association of SIRT1 gene variation with visceral obesity. Hum Genet 2008, 124:431-436.

114. Zillikens MC, van Meurs JBJ, Rivadeneira F, Amin N, Hofman A, Oostra Ba, Sijbrands EJ, Witteman JC, Pols HA, van Duijn CM, et al: SIRT1 genetic variation is related to BMI and risk of obesity. Diabetes 2009, 58:2828-2834.

115. Zhu J, Yong W, Wu X, Yu Y, Lv J, Liu C, Mao X, Zhu Y, Xu K, Han X, et at: Anti-inflammatory effect of resveratrol on TNF-a-induced MCP-1 expression in adipocytes. Biochem Biophys Res Commun 2008, 369:471-477.

116. Pfluger PT, Herranz D, Velasco-Miguel S, Serrano M, Tschop MH: Sirt1 protects against high-fat diet-induced metabolic damage. Proc Natl Acad Sci 2008, 105:9793-9798.

117. Fischer-Posovszky P, Kukulus V, Tews D, Unterkircher T, Debatin KM, Fulda S, Wabitsch M: Resveratrol regulates human adipocyte number and function in a Sirt1-dependent manner. Am J Clin Nutr 2010, 92:5-15.

118. Yoshizaki T, Milne JC, Imamura T, Schenk S, Sonoda N, Babendure JL, Lu JC, Smith JJ, Jirousek MR, Olefsky JM: Sirt1 exerts anti-inflammatory effect and improves insulin sensitivity in adipocytes. Mol Cell Biol 2009, 29:1363-1374.

119. Ramadori G, Lee CE, Bookout AL, Lee S, Williams KW, Anderson J, Elmquist JK, Coppari R: Brain SIRT1: anatomical distribution and regulation by energy availability. J Neurosci 2008, 28:9989-9996.

120. Cakir I, Perello M, Lansari O, Messier NJ, Vaslet CA, Nillni EA: Hypothalmaic SIRT1 regulates food intake in a rodent model system. PLOS ONE 2009, 4: e8322.

121. Ramadori G, Fujikawa T, Fukuda M, Anderson J, Morgan DA, Mostoslavsky R, Stuart RC, Perello M, Vianna CR, Nillni EA, et al: SIRT1 deacetylase in POMC neurons is required for homeostatic defenses against diet-induced obesity. Cell Metab 2010, 12.

122. Bereswill $S$, Muñoz M, Fischer A, Plicket R, Haag LM, Otto B, Kühl AA, Loddenkemper C, Göbel UB, Heimesaat MM: Anti-inflammatory effects of resveratrol, curcumin and simvastatin in acute small intestinal inflammation. PLOS One 2010, 5:e15099. 
123. Hofseth $\sqcup$, Singh UP, Singh NP, Nagarkatti M, Nagarkatti PS: Taming the beast within: resveratrol suppresses colitis and prevents colon cancer. Aging 2010, 2:183-184.

124. Larrosa M, Yanez-Gascon MJ, Selma MV, Gonzalez-Sarrias A, Toti S, Ceron JJ, Tomas-Bareran F, Dolara P, JC E: Effect of low dose of dietary resveratrol on colon microbiota, inflammation and tissue damage in a DSS-induced colitis rat model. J Agric Food Chem 2009, 57:2211-2220.

125. Cani PD, Delzenne NM: Involvement of the gut microbiota in the development of low grade inflammation associated with obesity: focus on this neglected partner. Acta Gastroenterol Belg 2010, 73:267-269.

126. Vanamala J, Tarver CC, Murano PS: Obesity-enhanced colon cancer: functional food compounds and their mechanisms of action. Curr Cancer Drug Targets 2008, 8:611-633.

127. Bernard CE, Gibbons SJ, Gomez-Pinilla PJ, Lurken MS, Schmalz PF, Roeder JL, Linden D, Cima RR, Dozois EJ, Larson DW, et al: Effect of age on the enteric nervous system of the human colon. Neurogastroenterol Motil 2009, 21:746-747.

128. Liu M, Kirchgessner AL: Agonist- and reflex-evoked internalization of metabotropic glutamate receptor 5 in enteric neurons. J Neurosci 2000, 20:3200-3205.

doi:10.1186/1479-5876-9-202

Cite this article as: Lakhan and Kirchgessner: Gut microbiota and sirtuins

in obesity-related inflammation and bowel dysfunction. Journal of

Translational Medicine 2011 9:202.

\section{Submit your next manuscript to BioMed Central and take full advantage of:}

- Convenient online submission

- Thorough peer review

- No space constraints or color figure charges

- Immediate publication on acceptance

- Inclusion in PubMed, CAS, Scopus and Google Scholar

- Research which is freely available for redistribution

Submit your manuscript at www.biomedcentral.com/submit 